\title{
Propagation Prediction Model for Land Mobile Communication in Microcellular Environment
}

\author{
J.A.Shrawankar \\ Asstt. Professor, Dept. of Electronics Engg. Shri \\ Ramdeobaba College of Engg. \& Management \\ Nagpur, India
}

\begin{abstract}
The propagation channel is the principal contributor to many of the problems and limitations that degrades the performance of mobile radio systems. One obvious example is multipath propagation which is a major characteristic of the mobile radio channels, which severely affects the performance of digital systems by reducing the carrier-to-interference ratio. Thus accurate characterization of the propagation channel is very important for cost effective planning of Land Mobile Communication System.

This paper presents a simplified technique for modeling radio propagation channel applicable for microcellular environment. Two propagation channel models have been considered. These are statistical theory based Suzuki Model and deterministic Uniform Theory of Diffraction (UTD) based Ray Tracing model, to predict received signal in the microcellular environment. The various statistical parameters such as, cumulative distribution function (cdf), level crossing rate (lcr) and average duration of fade (adf) were obtained from the total received signal to characterize the propagation channel using both the models. The comparison of these characteristics is summarized which indicates the good agreement of statistical and deterministic models.
\end{abstract}

\section{General Terms}

Radio wave propagation, Mobile communication

\section{Keywords}

Channel characterization, fading effect, mobile cellular communication, and wireless system

\section{INTRODUCTION}

In a move towards the objective of providing communications to and from any person or terminal irrespective of their locations, it is necessary to accommodate a wide variety of scenarios including motorways, urban and sub-urban areas, parks, rural areas and even locations remote from any center of population. To cope with these requirements it is necessary to tailor the size and shape of cells to suit the local geography and the amount of teletraffic envisaged. The diverse nature of the required cell shapes can be accommodated through the use of different antennas, transmitter location, power levels, and carrier frequencies.

The concept of microcell [1] however has been suggested as the best solution in heavily built-up areas. A microcell may have dimensions of only a few hundred meters with base transceivers mounted on the street lamp level. Each fixed base transceiver is adequate coverage there is a partial overlap. The extent of coverage from a given base can be adjusted by the use of different antenna patterns and heights and by controlling the transmitted power. Microcells have the advantages that delay spread are small and this, together with extensive frequency reuse, should allow high capacity, high data-rate systems to be developed.

\author{
K.D.Kulat, Ph.D \\ Professor, Dept. of Electronics Engg. \\ Visvesvaraya National Institute of Technology, \\ Nagpur, India
}

As the number of cellular phone subscribers has increased, it has been necessary to use smaller cells to increase system capacity. Currently in high subscriber density areas, and considering the future expansion in the system, the microcells with cell radius of $1 \mathrm{~km}$ or less is required. The reduction in cell size is accomplished by lowering the base station antenna so that they are near, or even below, that of the surrounding buildings. Over the distance of 1 or $2 \mathrm{~km}$ in an urban area, the street grid can be very regular, the buildings quite uniform in size, and the terrain variation small. With the use of low base station antennas, mobile signal propagation and the nature of the received signal at the subscriber unit in such an environment will depend strongly on the direction from the base station to the subscriber relative to the street grid.

Microcells are attracting much attention simply because they can accommodate more subscribers per unit service area than macrocells, also they permit access by low power portables. As suggested in [2,3], smaller cell coverage with lower antenna height and lower transmission power results in milder propagation characteristics. The smaller multipath delay spread and shallow fading imply and feasibility of broadband signal transmission without excessive counter-measure techniques against multpath fading.

The commercial success of cellular mobile radio has led to an intense interest among wireless engineers in understanding and predicting the radio propagation characteristics for outdoor and indoor wireless applications. In modern systems, the radio links are about 20 kilometers or less, the antennas that create the link lie near to or among the buildings or even inside the buildings, and the wavelength is small compared to the building dimensions. As a result, the channel characteristics are strongly influenced by the buildings as well as by vegetation and terrain. In this environment, signals propagate from one antenna to the other over multiple paths that involve the process of reflection and transmission at walls and by the ground and process of diffraction at building edges and terrain obstacles. The multipath nature of the propagation makes itself felt in a variety of ways that have challenged the inventiveness of communication engineers[4,5]. Each new concept for dealing with multipath, calls for an even deeper understanding of the characteristics of the radio channel. Path loss information is essential for determining the microcell coverage area for various systems $[6,7]$.

\section{PROPAGATION MODELS}

The prediction of path loss is a very important step in planning a mobile radio system and accurate prediction methods are needed to determine the parameters of a radio system that will provide efficient and reliable coverage of a specified service area. Existing prediction models differ in their applicability over different terrain and environmental conditions; some are useful to general applications, others are restricted to more specific situations. What is certain is that there is no one model which stands out as being ideally suited 
to all environments and careful assessment by the user is normally required. In this section comparison of statistical and deterministic model is presented with the help of their simulation results.

\subsection{Suzuki Model}

A statistical channel model based on Suzuki Process [8], where in the long-term variation of lognormal distributed local mean is superimposed on short-term fading modeled with classical "Rician" distribution is the best choice for land mobile systems. In the existing Suzuki model, the Doppler frequency and amplitude coefficients need to be fit with measured data. This model considers generalised incoming waves with randomly distributed spatial angles of arrivals and phases as proposed by T. Aulin [14]. It also considers the effect of speed of mobile and direction of travel thus relate more closely to the real-world propagation scenario.

Mathematically, the total received signal at any instant of time is of the form.

$$
\eta(t)=\xi_{1}(t)+\xi_{2}(\mathrm{t})
$$

Where $\xi_{1}(t)$ represents the classical Rician distributed shortterm fading signal and is given by

$$
\xi_{1}(t)=E_{0}(t)+E_{1}(t)+\sum_{n=2}^{N-1} E_{n}(\mathrm{t})
$$

Where $E_{0}(t) \& E_{1}(t)$ are the coherent LOS and ground reflected components respectively and $E_{n}$ is the $n^{\text {th }}$ noncoherent multipath component (reflected, diffracted or combination of both).

A typical received multipath signal is of the form

$E n(t)=A_{n} * C_{n} * e^{-j\left(k w_{n} t+\phi_{n}\right)}$

In which $A_{n}$ account for the attenuation factor due to the length, $\mathrm{C}_{\mathrm{n}}$ model the complex dyadic diffraction/reflection (or product of the two) coefficient and $\phi_{\mathrm{n}}$ is the phase change, which are uniformly distributed random variables. Finally $\mathrm{k}$ is the wave number and $\omega_{\mathrm{n}}$ is the angular frequency shift due to the movement of receiver, which is given by

$w_{n}=\frac{v_{m}}{\lambda} \cos \left(\gamma-\alpha_{n}\right) \cos \left(\beta_{n}\right)$

In above equation a typical $\mathrm{n}^{\text {th }}$ incoming multipath wave at the receiver with an azimuth angle of $\alpha_{n}$ in the range of $(0,2 \pi)$ and an elevation angle of $\beta_{n}$ in the range of $(0, \pi / 2)$ is considered for the simulation. The receiver is assumed to be moving with a velocity of $\mathrm{v}_{\mathrm{m}}$ in the $\mathrm{x}-\mathrm{y}$ plane in a direction making an angle $\gamma$ to the $x$-axis.

The novelty of the model appears from the definitions of the above parameters based on the propagation theory. The log normal distributed shadowing effect shown in (1) with mean $\mathrm{m}$ and variance $\mathrm{s}$ is given by

$\xi_{2}(t)=e^{(s * \rho+m)}$

Where, $\rho$ is a uniformly distributed random variable lying the range of $[-1,1]$.

\subsection{UTD based Ray tracing model}

A three dimensional micro-cellular communications propagation channel model based on UTD and Ray-tracing technique is presented in this section. This with little modification will be applicable to a general city scene with various distributions of buildings, streets, trees and open areas. The advantages of this model include versatility, the ability to provide good understanding of the physical propagation mechanism and ability to predict the receive signal accurately.

The deterministic UTD based ray tracing model considers the geometry of the problem along with the electromagnetic properties of the physical environment and describes the propagation phenomena such as reflection and diffraction by the multipath components $[9,10]$. A typical microcellular site scenario as shown in figure 1 is considered for simulation whose geometry is provided in Table 1, with building length $\mathrm{L}$, width $\mathrm{W}$, and height $\mathrm{H}$. The building walls are assumed as perfect conductors and "smoothed out" flat surfaces. The total received signal at mobile receiver on move with reference to the base station located at $(0,0,0)$, at any instant of time is the vectorial addition of all the multipath components, which can be calculated as described below.<smiles>[Y]C([1H])[13CH3]</smiles>

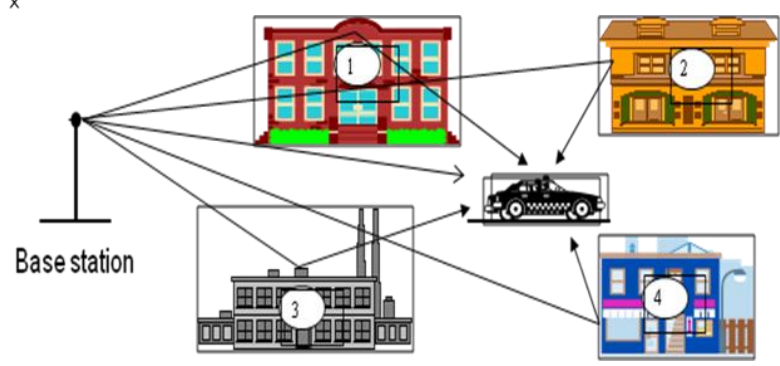

Fig 1: Typical layout considered for multipath propagation

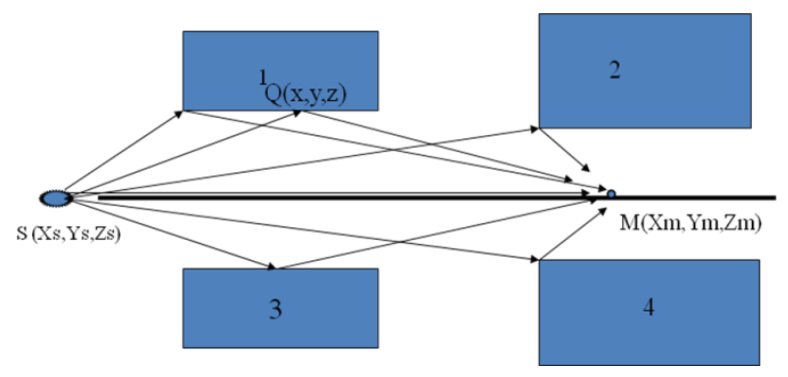

Fig 2: Plane views of fig1 with various multipath signals

Table 1. Building data considered for simulation.

\begin{tabular}{|l|c|c|c|c|}
\hline $\begin{array}{l}\text { Building } \\
\text { No. }\end{array}$ & $\mathbf{L}(\mathbf{m})$ & $\mathbf{W}(\mathbf{m})$ & $\mathbf{H}(\mathbf{m})$ & Building Centre \\
\hline 1 & 120 & 20 & 20 & $(-17.5,80,0)$ \\
\hline 2 & 90 & 25 & 18 & $(-17.5,200,0)$ \\
\hline 3 & 80 & 30 & 20 & $(19.5,90,0)$ \\
\hline 4 & 110 & 24 & 19 & $(19.5,195,0)$ \\
\hline
\end{tabular}


At the mobile receiver the line of sight (LOS) field is given by

$E_{\text {los }}=\frac{E_{i}}{\sqrt{d_{s m}}}$

Where $E_{i}$ represents incident signal and $d_{s m}$ is the distance between the transmitter and the mobile receiver.

For a reflection or diffraction at a specular point, the incident wave $E_{i}$ gives rise to a reflected or diffracted wave at the mobile receiver given by $E_{r, d}(M)$.

Where

$E_{r, d}(M)=E_{i}(Q) * H(Q, M) * e^{j k\left(s+s^{\prime}\right)}$

$H(Q, M)=\mathrm{R} \cdot \operatorname{Ar}(\mathrm{s})$ for reflection

D. Ad(s) for diffraction

$\mathrm{R}=$ reflection coefficient, $\mathrm{D}=$ diffraction coefficient,

$\operatorname{Ar}(\mathrm{s})=$ spreading factor for a reflection from surface,

$\operatorname{Ad}(\mathrm{s})=$ spreading factor for a diffraction at an edge, $\mathrm{k}=$ propagation constant, $\mathrm{s}=$ distance from source to specular point, $\mathrm{s}^{\prime}=$ distance from specular point to mobile receiver. All these coefficients are evaluated as specified in [11].

The total received field at any position of mobile receiver is calculated by vectorial addition of all the multipath contribution (like direct Los, reflected field and diffracted field) which is given by

$\vec{E}_{\text {total }}=\vec{E}_{\text {los }}+\vec{E}_{r}+\vec{E}_{d}$

\section{RESULTS AND CONCLUSSION}

The total received signal in a typical urban microcellular scenario is calculated using deterministic UTD based Ray tracing techniques. The first and second order statistical parameters are calculated and compared with the statistical theory based Suzuki model.

The attenuation caused by multipath propagation and shadowing effects is shown in figure 3 as a function of distance of travel. If a certain minimum signal level (threshold) is needed for the acceptable communication performance, complementary cumulative distribution function (ccdf) at any threshold gives the probility that signal level lies above the threshold value. The ccdf curve obtained from UTD based model is shown in figure 4 . Figure 5 shows the variation of level crossing rate lcr as a function of threshold. The received signal experience periods of sufficient signal strength or "non-fade intervals", during which receiver can works reliably at low bit error rate and sufficient signal strength or "fading interval" during which the receiver may not function satisfactorily. Figure 6 shows the average fade duration (afd) as a function of threshold level. The adf curve helps to determine the most likely number of bits that may be lost during the fade. The characteristics lcr and adf are known as second-order statistical characteristics since they depends on both observation time and velocity of mobile. The comparison of the various characteristics indicated in table 2 shows that both the models are are well in agreement with each other.
Table 2. Comparison of the radio channel propagation characteristics.

\begin{tabular}{|l|l|l|}
\hline $\begin{array}{l}\text { Propagation Characteristics } \\
\text { (at }-\mathbf{1 5} \text { dB threshold) }\end{array}$ & $\begin{array}{l}\text { Suzuki } \\
\text { Model }\end{array}$ & $\begin{array}{l}\text { UTD } \\
\text { based }\end{array}$ \\
\hline Prob. Density function & 0.05 & 0.039 \\
\hline $\begin{array}{l}\text { Cumulative distribution } \\
\text { function }\end{array}$ & 0.7 & 0.68 \\
\hline Level crossing rate & 0.15 & 0.155 \\
\hline Average fade duration & 0.45 & 0.53 \\
\hline
\end{tabular}

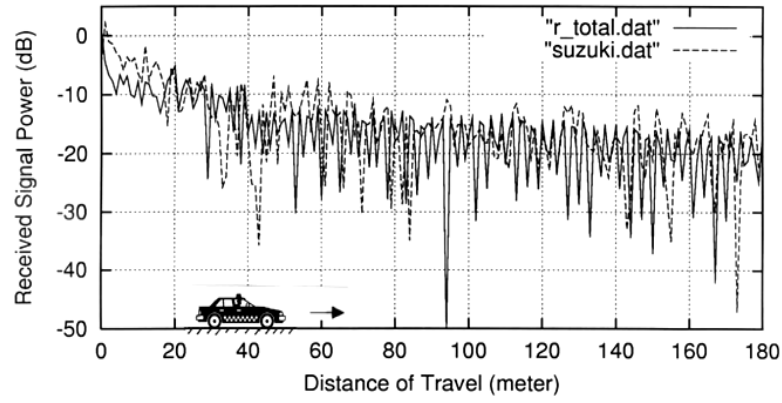

Fig3: Comparison of received signal envelop

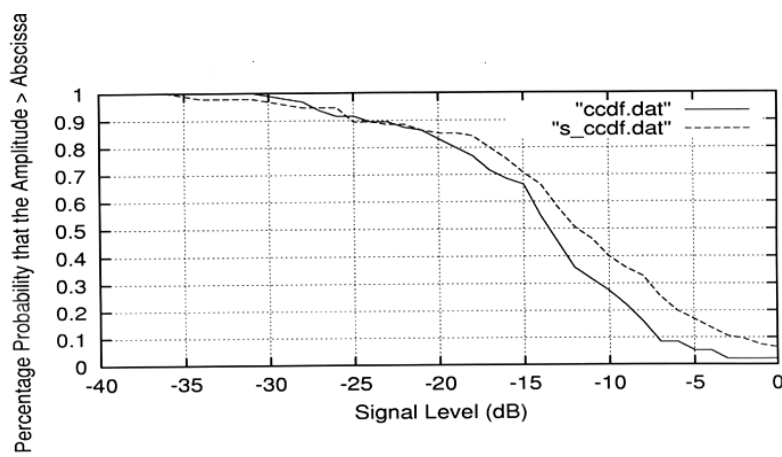

Fig 4: Comparison of ccdf of received signal envelop

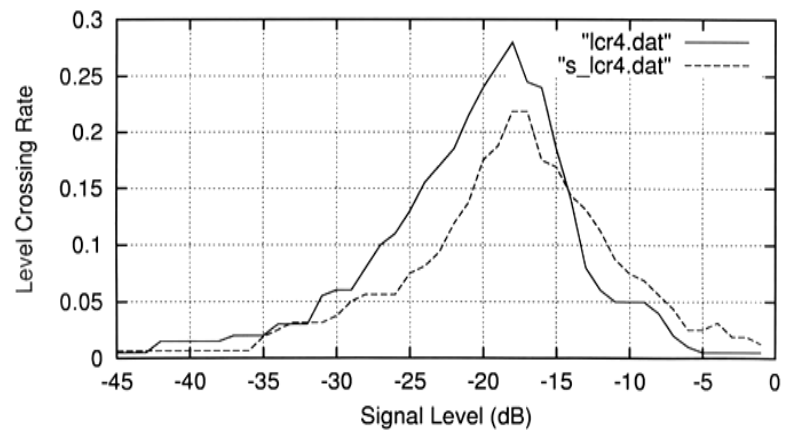

Fig 5: Comparison of lcr of received signal envelop 


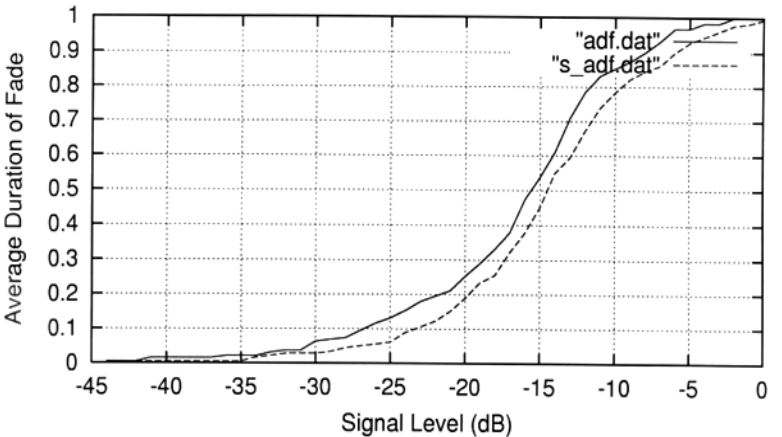

Fig 6: Comparison of adf of received signal envelop

\section{REFERENCES}

[1] T. S. Rappaport, "Wireless communications : principles and practice" PHI publication,2003.

[2] Lee William C.Y., “ Mobile Communications: Design Fundamentals” John Wiley \& sons 1993

[3] Parson J.D., “ The mobile radio propagation channel” Pentech press, 1994

[4] Sarkar T.K. et. al. " A survey of various propagation models for mobile communication" IEEE Antenna and Propagation Magazine, Vol. 45, No.3, June 2003, pp 51-82.

[5] M.F.Iskander ,Z.Yun, "Propagation Prediction Models for Wireless Communication Systems.” IEEE
Transaction on Microwave Thory and Rechniques, MTT50,3,2002,pp. 662-673

[6] Andersen J.B. , Rappaport T.S., Susumu Yoshida ," Propagation measurements and models for wireless communication channels" ,IEEE Communication Magazine Jan. 1995 , pp 42-49.

[7] J.A.Shrawankar \& Dr. K.D.Kulat "A study of the influence of path loss and Short term Fading on the Performance of Mobile Radio System", International Journal of Computer Applications, Volume 70, No. 27, May 2013, ISSN 0975-8887, pp 01-04.

[8] Suzuki H.," A Statistical Model for urban radio Propagation," IEEE Tran. Comm., vol-COMM-25,no7,pp. 673-680,(1977)

[9] Kouyoumjian R.G. and Pathak P.H.,"A Uniform Geometrical Theory of Diffraction for an edge in a perfectly conducting surfaces," Proc.IEEE Vol62,pp.1448-1461,(1974)

[10] Rusch W.V.T and Soranson O., " On Determining if a Specular point exits," IEEE Tran. AP ,vol-27,no.1, pp.99-101,Jan. (1979).

[11] Tan S.Y. and Tan H.S.," A Theory for propagation Path Loss Characteristics in a city street grid ," IEEE Tran. Electromagnetic Compt. Vol.37, pp.333-342,Aug. (1995) 